\title{
Esophageal perforation: a delayed complication following traumatic spinal cord injury. Case report
}

\author{
$\mathrm{S}$ Colachis III MD, ${ }^{1} \mathrm{~K}$ D Murray $\mathrm{MD}^{2}$ \\ ${ }^{1}$ Assistant Professor, Department of Physical Medicine and Rehabilitation and Director, \\ Spinal Cord Injury Rehabilitation, The Ohio State University, Dodd Hall, 480 West Ninth \\ Avenue, Columbus, Ohio 43210, USA $;{ }^{2}$ Assistant Professor, Division of Thoracic and \\ Cardiovascular Surgery, The Ohio State University, Columbus, Ohio, USA.
}

\begin{abstract}
Esophageal perforation is an uncommon but serious complication of penetrating injuries to the neck and chest. A case of delayed esophageal perforation in a patient with traumatic spinal cord injury is presented. The pathophysiology, diagnosis, and treatment of this potentially fatal complication are discussed.
\end{abstract}

Key words: spinal cord injury; esophageal perforation; paraplegia; gunshot wound.

\section{Introduction}

Esophageal perforation is a serious complication of penetrating wounds to the neck and chest. In untreated cases, mortality approaches $100 \% .{ }^{1}$ Early diagnosis is essential for effective management as mortality increases from between $8 \%$ and $33 \%$ in ruptures diagnosed within several hours of injury, to between $40 \%$ and $100 \%$ in cases where the diagnosis exceeds 24 hours from the time of perforation, depending upon the series cited. ${ }^{2-8}$ The non specific findings and rapid catastrophic sequelae in patients with esophageal injury contribute to the high mortality. ${ }^{6} \mathrm{~A}$ high index of suspicion is required in all cases of penetrating injury to the chest and neck.

A following case of delayed perforation of the esophagus illustrates the difficulty of diagnosis in a spinal cord injured patient .

\section{Case report}

On October 1, 1988, a 31 year old man sustained a gunshot wound (.38 caliber) to the neck at close range resulting in immediate T3 complete paraplegia. Diagnostic work-up at that time demonstrated comminuted fractures of $\mathrm{T} 3$ and $\mathrm{T} 4$ vertebrae and bilateral lung contusions with hydrothorax. The bullet fragment was situated within the superior mediastinum. There was no evidence of pneumothorax or subcutan- eous emphysema on admission. An arch aortogram and esophagram with video fluoroscopy performed on admission were normal. Mediastinal widening was seen on admission chest radiographs; this finding was attributed to mediastinal venous injury. Esophagoscopy was not performed because of the patient's unstable thoracic spine fracture.

The patient's hydrothoraces were drained with bilateral chest tubes without difficulty and the chest tubes were removed 6 days after admission. Small pneumothoraces present after chest tube placement resolved shortly after the tubes were removed. He was started on oral intake after resolution of an actute ileus approximately 10 days after injury. A computed axial tomography (CAT) scan of the thorax performed on October 10, 1988 showed burst fractures of T3 and T4. Large paravertebral and mediastinal hematomas were present but there was no evidence of pneumomediastinum at that time. He underwent fusion and stabilization with Luque rods and Drummond wiring from C7 to T7 on October 24, 1988. His hospital course was also complicated by pneumonia.

The patient was transferred to the spinal cord injury rehabilitation service 4 weeks after injury. He was eating at that time without difficulty. Shortly after admission, he developed persistent fevers and leukocytosis without associated signs or symptoms 
of systemic disease. Urine, sputum, and blood cultures were all negative. A chest $\mathrm{x}$-ray demonstrated resolution of the prior pneumonia, but suggested the presence of a soft tissue density in the right lung. In view of persistent fevers and leukocytosis, despite a paucity of symptoms, CAT scans of the neck and chest were obtained on November 4, 1988. Multiple air-fluid levels were observed in the mediastinum (Fig 1). The patient was started on intravenous antibiotics empirically and a repeat esophagram was performed (Fig 2) which revealed an esophageal tear with linkage of barium and air into the superior mediastinum.

$\mathrm{He}$ subsequently underwent mediastinal exploration with surgical drainage on November 11, 1988, and thoracotomy with wide drainage of the mediastinum and esophagus on December 5, 1988. Hyperalimentation was employed until gastrostomy and feeding jejunostomy tubes were placed. He was transferred for continued spinal cord injury rehabilitation on January 23, 1989, The esophageal leak finally healed on February 21, 1989.

\section{Discussion}

Esophageal perforation can be a lethal complication of penetrating chest or neck trauma. Despite advances in early diagnosis and management, mortality continues to be reported as over $20 \% .^{3}$ In untreated cases, the condition is considered fatal. ${ }^{1}$ Factors adversely affecting outcome have included location and severity of the injury, ${ }^{8-10}$ preexisting esophageal disease ${ }^{8}$ associated injures, ${ }^{6.10}$ and delay in diagnosis and treatment of greater than 12 to 24 hours from the time of injury. ${ }^{3.5,8,11.12}$ Delay in diagnosis and treatment is a most ominous factor with mortality exceeding $50 \%$ in cases treated greater than 24 hours after injury. 2,6

The morbidity and mortality are significantly less for cervical esophageal injuries

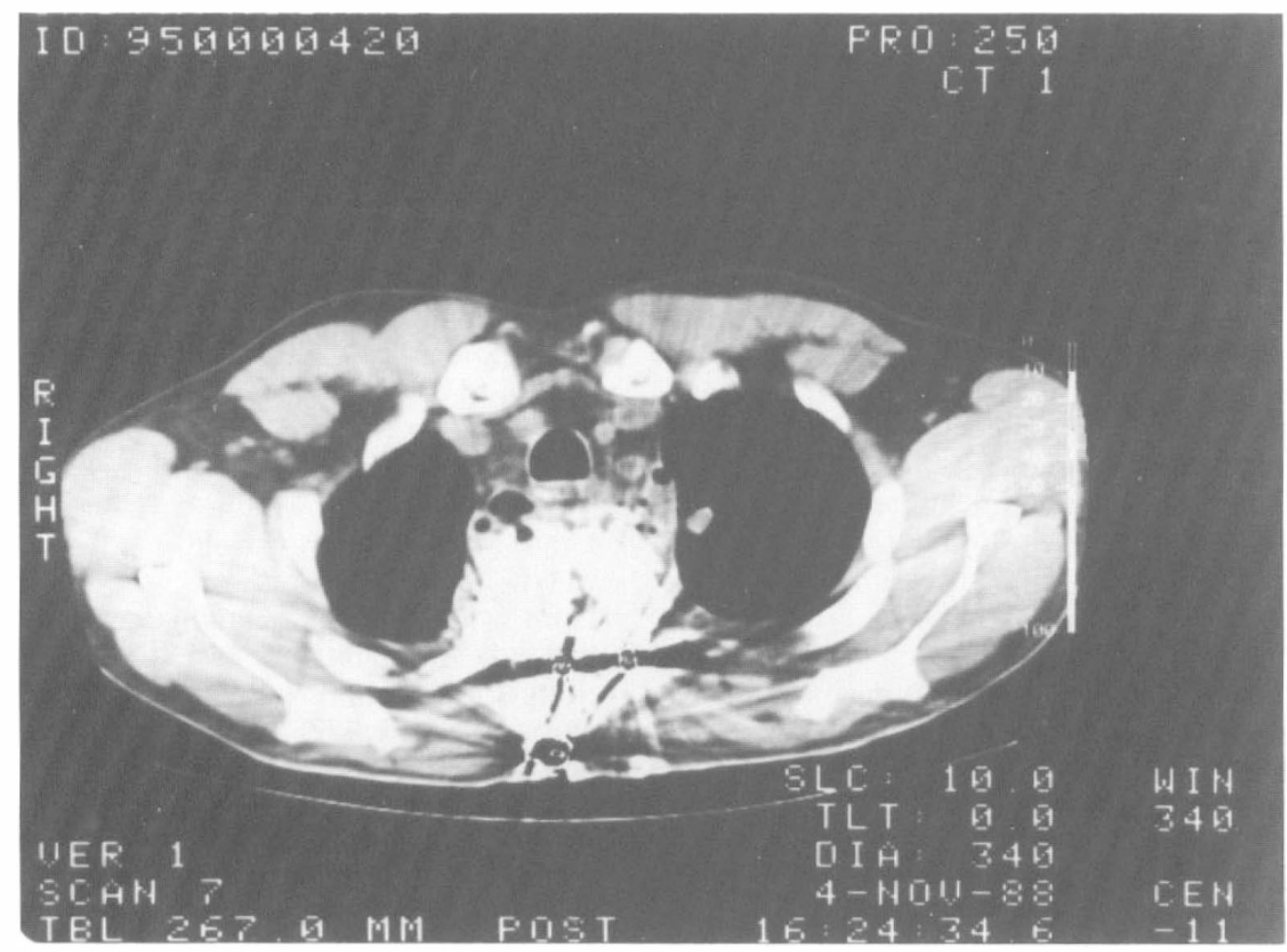

Figure 1 Computed axial tomography (CAT) scan of the upper chest in the region of the vertebral fracture demonstrating air-fluid levels in the mediastinum. 


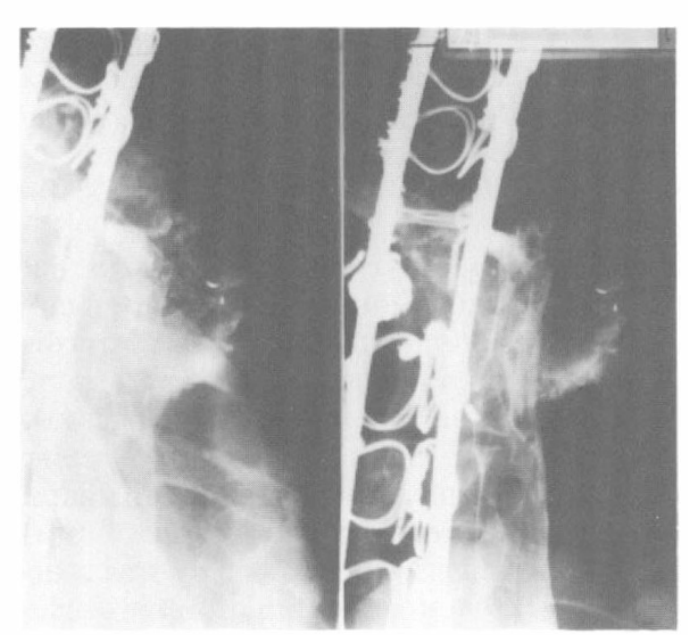

Figure 2 Esophagram showing leakage of contrast material and air into the superior mediastinum (oblique view).

than for thoracic perforations. ${ }^{2-4.9 .12 .13}$ Injuries to the thoracic esophagus are often associated with serious injury to other vital structures, ${ }^{4}$ and signs and symptoms can be non specific. Thoracic esophageal contents can rapidly result in widespread mediastinal contamination, serious mediastinitis, abscess formation, and sepsis. ${ }^{3.5 .6 .9 .11}$ Thoracic esophageal perforations can result in esophageal fistulas, ${ }^{4.7}$ empyema, ${ }^{4}$ arterial erosions, ${ }^{6}$ or esophageal stricture, and may necessitate esophageal resection. ${ }^{2.5}$

The high morbidity and mortality associated with esophageal perforations have led many surgeons to adopt a policy of mandatory exploration of all neck wounds penetrating the platysma muscle. ${ }^{1+.15}$ This approach has been reported to result in a high number of negative explorations: ${ }^{16-18}$ therefore, others have employed selective non operative management in cases of penetrating neck injuries. ${ }^{16.17 .19-21}$ Selective management includes careful patient selection, close observation, and use of invasive diagnostic studies. ${ }^{19.20 .22}$ Initial management of neck wounds remains controversial. ${ }^{15.17 .20}$

Signs and symptoms of esophageal perforation are often non specific and associated injuries may mask the clinical findings of rupture. ${ }^{4.6 .17}$ Patients with an esophageal injury may present with neck or chest pain, dyspnea, dysphagia, hoarseness, choking, hematemesis, dysphonia, or generalized malaise. ${ }^{2-4,7,10,13}$ Cardiovascular collapse can occur, depending upon the location of injury, the severity, and associated trauma. Various clinical signs helpful in the diagnosis include expanding hematoma, ${ }^{18}$ subcutaneous crepitus or emphysema, ${ }^{3.10}$ nasogastric aspiration of blood, mediastinal crunch sound of Hamman, and unstable clinical condition. ${ }^{7}$ Other non specifc findings include fever and leukocytosis. ${ }^{10}$

Radiographic findings useful in the diagnosis of esophageal perforations involve demonstration of subcutaneous emphysema, pneumomediastinum, pneumothorax, and mediastinal widening. ${ }^{3,4,7,10.13,18}$ Plain film chest radiographs may reveal a pulmonary contusion, foreign body, rib fracture, or effusion. ${ }^{4}$ Findings such as these are common in penetrating injuries to the chest. Definitive diagnosis is made by esophagram, esophagoscopy, and surgical exploration. Unfortunately, false negative results have been reported in all these diagnostic procedures employed. . $^{\text {7.17.21 }}$

The specific management of esophageal perforations remains controversial. ${ }^{2,11}$ The experience of most authors reviewed strongly supports early operative management, including primary surgical repair and drainage. ${ }^{2.36 .7 .9 .10 .20}$ This is especially true in cases of thoracic esophageal perforations. ${ }^{3.4 .9}$ If injury is unrecognized early, surgical repair may no longer remain an option, ${ }^{6.8}$ and associated morbidity and mortality increase significantly. Although conservative management has been reported in selected cases, ${ }^{1.2 .11 .23}$ surgical management of thoracic esophageal perforations from penetrating injuries is most commonly advocated. ${ }^{2.3 .6 .7 .9 .10 .20}$

In cases of thoracic esophageal perforation diagnosed within several hours of injury, surgical management consists of thoracotomy, primary repair, and drainage. . $^{2.3 .6-10}$ Esophageal exclusion procedures and reinforcement of the repair with pleural or flaps, muscle bundles, or fat grafts have also been employed. ${ }^{3,4.9 .10 .2+}$ Failure of primary repair can occur and is of serious concern. . $^{9} 10.12$ This complication increases with delayed intervention. ${ }^{1}$ Surgical management is supplemented with broad spectrum antibiotics, 
nasogastric decompression, and parenteral nutrition. ${ }^{1,7,13}$ Treatment of esophageal perforations with delayed presentation or diagnosis entails thoracotomy with wide mediastinal drainage. ${ }^{8}$ Effective drainage, broad spectrum antibiotics, and adequate nutrition are essential in such cases. ${ }^{5}$ Decompression gastrostomy and feeding jejunostomy are commonly employed in the management of these cases. ${ }^{8,24}$

The current case illustrates the difficulty in diagnosis of an esophageal perforation in a patient with a spinal cord injury. Chest or back pain commonly observed in thoracic esophageal perforations would not necessarily be appreciated in T3 complete paraplegia, as was the case with this individual. In addition, many of the other symptoms of esophageal injury (eg dysphonia, dysphagia, choking, hematemesis, malaise, etc) were absent. The patient had no obvious clinical signs of esophageal perforation (eg subcutaneous crepitus, crunch sign of Hamman, etc) or systemic illness except fever, and all cultures were negative. It is well known that spinal cord injuries above T4 to T6 may result in abnormalities of thermal regulation which manifest as fever. ${ }^{25}$ The patient had been on steroids which could account, at least in part, for the leukocytosis present. ${ }^{26}$ Multiple surgical wounds present were potential sites of infection and confused the clinical picture. It was the radiographic abnormalities which first suggested the possibility of esophageal perforation and confirmed the suspicion.

The paucity of clinical findings in the present case made exact determination of the time of rupture difficult. The bullet fragment or percussion wave from the close range of fire initially injured the esophagus and perforation occurred sometime after resumption of oral intake a few days prior to diagnosis. It is unlikely that the gunshot wound resulted in esophageal perforation at the time of injury. Such an occurrence would have undoubtedly resulted in death, as identification of perforation was 5 weeks after injury and no surgical drainage was peformed during that period. At the time of injury, subcutaneous crepitus and the crunch sound of Hamman were absent, and nasogastric drainage failed to identify any gastrointestinal blood. Initial radiographic evaluation showed bilateral lung hematomas without pneumothorax or pneumomediastinum; chest tube drainage was performed for hematoma evacuation only. Mediastinal widening was present on initial films and was attributed to mediastinal venous injury. This finding remained stable and decreased throughout hospitalization. During the following weeks of hospitalization repeat radioagraphs including CAT scanning of the thorax performed 10 days after injury failed to demonstrate pneumomediastinum.

Although esophagoscopy is employed at this institution in the initial evaluation of patients with penetrating wounds to the neck, this procedure was not performed due to the unstable fracture. An esophagram with video studies, however, was normal; all esophagrams performed subsequent to the perforation were positive until the leak healed.

In view of the difficulty in determining the time of esophageal perforation, surgical management was directed at wide mediastinal debridement and drainage, gastric decompression, broad spectrum antibiotic coverage, and parenteral nutrition. The patient's esophagus healed, and he completed rehabilitation.

\section{References}

1 Lyons WS, Seremetis MG, deGuzman VC, Peabody JW (1978) Ruptures and perforations of the esophagus: the case for conservative supportive management. Ann Thorac Surg 25: 346-350.

2 Bladergroen MR, Lowe JE, Postlethwait RW (1986) Diagnosis and recommended management of esophageal perforation and rupture. Ann Thorac Surg 42: 235-239.

3 Cohn HE, Hubbard A, Patton G (1989) Management of esophageal injuries. Ann Thorac Surg 48: 309-314.

4 Defore WW, Mattox KL, Hansen HA, Garcia-Rinaldi R, Beall AC, DeBakey ME (1977) Surgical management of penetrating injuries of the esophagus. Am J Surg 134: 734-738.

5 Mayer JE, Murray CA, Varco RL (1977) The treatment of esophageal perforation with delayed recognition and continuing sepsis. Ann Thorac Surg 23: 568-573. 
6 Noon GP, Beall AC, DeBakey ME (1968) Surgical management of penetrating esophageal injuries. $J$ Trauma 8: 458-464.

7 Pass LJ, LeNarz LA, Schreiber JT, Estrera AS (1987) Management of esophageal gunshot wounds. Ann Thorac Surg 44: 253-256.

8 Richardson JD, Martin LF, Borzotta AP, Polk HC (1985) Unifying concepts in treatment of esophageal leaks. Am J Surg 149: 157-62.

9 Brewer LA, Carter R, Mulder GA, Stiles QR (1986) Options in the management of perforations of the esophagus. Am J Surg 152: 62-69.

10 Popovsky J (1984) Perforations of the esophagus from gunshot wounds. J Trauma 24: 337-339.

11 Cameron JL, Kieffer RF, Hendrix TR, Mehigan DG, Baker RR (1979) Selective nonoperative management of contained intrathoracic esophageal disruptions. Ann Thorac Surg 27: 404-408.

12 Symbas PN, Tyras DH, Hatcher CR, Perry B (1972) Penetrating wounds of the esophagus. Ann Thorac Surg 13: 552-558.

13 Oparah SS, Mandal AK (1979) Operative management of penetrating wounds of the chest in civilian practice: review of indications in 125 consecutive patients. J Thorac Cardiovasc Surg 77: 162-168.

14 Ashworth, C, Williams, LF, Byrne JJ (1971) Penetrating wounds of the neck. Am J Surg 121: 387-391.

15 Bishara RA, Pasch, AR, Douglas DD, Schuler JJ, Lim LT, Flanigan DP (1986) The necessity of mandatory exploration of penetrating zone II neck injuries. Surgery 4: 655-660.

16 Metzdorff MT, Lowe DK (1984) Operation or observation for penetrating neck wounds? A retrospective analysis. Am J Surg 147: 646-649.

17 Noyes LD, McSwain NE, Markowitz IP (1986) Panendoscopy with arteriography versus mandatory exploration of penetrating wounds of the neck. Ann Surg 204: 21-31.

18 Weigelt JA, Thal ER, Synder WH, Fry RE, Meier DE, Kilman WJ (1987) Diagnosis of penetrating cervical esophageal injuries. Am J Surg 154: 619-622.

19 O'Donnell VA, Atik M, Pick RA (1979) Evaluation and management of penetrating wounds of the neck: the role of emergency angiography. Am J Surg 138: 309-313.

20 Ordog GJ, Albin D, Wasserberger J, Schlater TL, Balasubramaniam S (1985) One hundred and ten bullet wounds to the neck. J Trauma 25: 238-46.

21 Wood J, Fabian TC, Mangiante EC (1989) Penetrating neck injuries: recommendations for selective management. J Trauma 29: 602-605.

22 Richardson JD, Flint LM, Snow NJ, Gray LA, Trinkle JK (1981) Management of transmediastinal gunshot wounds. Surgery 90: 671-676.

23 Mengoli LR, Klassen KP (1965) Conservative management of esophageal perforation. Arch Surg 91: $238-240$.

24 Urschel HC, Razzuk MA, Wood RE, Galbraith N, Pockey M, Paulson DL (1974) Improved management of esophageal perforation: exclusion and diversion in continuity. Ann Surg 179: 587-591.

25 Guttmann L, Silver J, Wyndham CH (1958) Thermoregulation in spinal man. J Physiol 142: 406-419.

26 Bishop CR, Athens JW, Boggs DR, Warner HR, Cartwright GE, Winthrobe MM (1968) Leukokinetic studies XIII. A non-steady-state kinetic evaluation of the mechanism of cortisone-induced granulocytosis. $J$ Clin Invest 47: 249-260. 